\title{
Abnormal Structural Luteolysis in Ovaries of the Senescence Accelerated Mouse (SAM): Expression of Fas Ligand/Fas- Mediated Apoptosis Signaling Molecules in Luteal Cells
}

\author{
Minako KISO'), Noboru MANABE ${ }^{1)}$, Kohji KOMATSU1), Munetake SHIMABE ${ }^{1)}$ and \\ Hajime MIYAMOTO ${ }^{1)}$
}

1) Unit of Anatomy and Cell Biology, Department of Animal Sciences, Kyoto University, Kyoto 606-8502, Japan

\begin{abstract}
Senescence accelerated mouse-prone (SAMP) mice with a shortened life span show accelerated changes in many of the signs of aging and a shorter reproductive life span than SAMresistant (SAMR) controls. We previously showed that functional regression (progesterone dissimilation) occurs in abnormally accumulated luteal bodies (aaLBs) of SAMP mice, but structural regression of luteal cells in aaLB is inhibited. A deficiency of luteal cell apoptosis causes the abnormal accumulation of LBs in SAMP ovaries. In the present study, to show the abnormality of Fas ligand (FasL)/Fas-mediated apoptosis signal transducing factors in the aaLBs of the SAMP ovaries, we assessed the changes in the expression of FasL, Fas, caspase- 8 and caspase- 3 mRNAs by reverse transcription-polymerase chain reaction, and in the expression and localization of FasL, Fas and activated caspase- 3 proteins by Western blotting and immunohistochemistry, respectively, during the estrus cycle/luteolysis. These mRNAs and proteins were expressed in normal LBs of both SAMP and SAMR ovaries, but not at all or only in trace amounts in aaLBs of SAMP, indicating that structural regression is inhibited by blockage of the expression of these transducing factors in luteal cells of aaLBs in SAMP mice.
\end{abstract}

Key words: Apoptosis, Fas ligand/Fas system, Intracellular signal transduction, Luteolysis, Senescence accelerated mouse (SAM)

(J. Reprod. Dev. 49: 457-463, 2003)

$\mathbf{T}$ he senescence accelerated mouse (SAM) strain, which is an inbred strain of AKR mice, was bred and established in Kyoto University by Takeda et al. [1, 2] and is a useful animal model for studying senescence. SAM-prone (SAMP) mice show accelerated changes in many of the signs of aging including a moderate to severe loss of activity, hair loss and lack of hair glossiness, skin coarseness, periophthalmic lesions, increased lordokyphosis of the spine and a shortened life span as compared with SAM-resistant (SAMR)

Accepted for publication: August 6, 2003

Correspondence: N. MANABE (e-mail: manabe@kais.kyotou.ac.jp) mice, which is the control strain that ages normally. Although physiological, pathological and biochemical changes associated with aging in SAMP mice with advancing age have been studied by many investigators [3], there have been insufficient data on reproductive properties in SAM mice. We previously compared male $[4,5]$ and female reproductive properties and the early embryonic development of SAMP mice with those of SAMR mice [6, 7]. During the investigations on the reproductive properties of SAM mice, we found abnormally accumulated luteal bodies (aaLBs) in the ovaries of SAMP mice [8]. During the estrous cycle, no differences between the kinetics of $17 \beta$ - 
estradiol (E2) and progesterone (P4) in peripheral blood were observed in SAMP and SAMR mice, indicating that there is no difference in female hormonal regulation between the two strains [6-8]. In luteal cells of aaLBs of SAMP mice, a high level of activity of $20 \alpha$-hydroxysteroid dehydrogenase

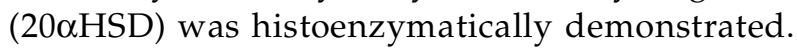
P4 is catabolized to 20 $\alpha$-hydroxyprogesterone, a physiologically inactive form, by $20 \alpha$ HSD in luteal cells of rodents. Our histo-enzymatic findings indicate that functional regression, a decrease in P4 production, occurs in the luteal cells of aaLBs in SAMP mice. Nevertheless, no apoptotic cells were detected histochemically in aaLBs of SAMP ovaries, but many positive cells were detected in the LBs of SAMR ovaries and in some normal LBs of SAMP ovaries. These histochemical and biochemical findings confirm that an arrest of morphological regression (deficiency of apoptotic cell death) occurs in the luteal cells of aaLBs in SAMP mice [8]. An increase in Fas (a cell death-receptor)-positive luteal cells as assessed by immunohistochemistry was observed in granulosa-luteal cells during luteolysis in murine ovaries $[9,10]$. Moreover, Fas ligand (FasL) and/or anti-Fas-antibody induce apoptotic cell death in cultured luteal cells in mice. Homozygous lpr mice, which have a non-functional Fas [11], and homozygous gld mice, which lack a functional FasL, had an irregular luteal phase. The FasL/Fas system plays crucial roles in the regulation of luteolysis in murine ovaries [8-13]. The FasL/Fas-dependent apoptosis was mediated by the activation of an intracellular caspase cascade (caspase-8, caspase-3 etc.) [14].

In the present study, to elucidate the abnormalities of FasL/Fas-mediated apoptosis signal-inducing factors in luteal cells of aaLBs in SAMP mice, we compared the expression of mRNAs of apoptosis-related proteins (FasL, Fas, caspase- 8 and caspase- 3 ) in normal LBs with those in aaLBs of SAMP ovaries by the reverse transcription-polymerase chain reaction (RT-PCR) technique. We then examined the changes in the expression and localization of FasL, Fas and activated caspase-3 proteins, assessed by Western immunoblotting and immunohistochemistry, respectively, during luteal regression of these LBs.

\section{Materials and Methods}

\section{Animals and ovarian tissue sampling}

As described in our previous reports [6-8], mature female mice (20 to 30 weeks old) of the SAMP (SAMP1//FAP) and SAMR (SAMR1/FAP) strains, with more than two consecutive 4-dayestrus cycles determined by daily examination of vaginal cytology were used. All animals were housed in an air-conditioned room $(22 \pm 2 \mathrm{C}, \mathrm{RH} 60$ $\pm 5 \%)$ under controlled lighting conditions (12 L/12 D) and given a standard diet (NMF; Oriental Yeast Co., Tokyo, Japan) and tap water ad libitum. They received humane care as outlined in the "Guide for the Care and Use of Laboratory Animals" (Kyoto University Animal Care Committee according to NIH \#86-23; revised 1999). The animals were sacrificed under deep ether anesthesia in the afternoon (14:00) at proestrus, estrus, metestrus and diestrus (P, E, M and D, respectively), and then the ovaries were rapidly removed, weighed and cut. For histology and immunohistochemistry, some of the ovaries were fixed in $10 \%(\mathrm{v} / \mathrm{v})$ phosphate buffered formalin, $\mathrm{pH}$ 7.4. For biochemical analyses, some of the tissue was frozen in liquid nitrogen.

Histology and immunohistochemistry for FasL, Fas, activated caspase- 3 and ssDNA

Fixed ovarian tissues were dehydrated through a graded ethanol series and embedded in paraffin. Serial sections ( $3 \mu \mathrm{m}$ thick) were cut from the median part of each ovary, mounted on glass slides coated with 3-aminopropyltrimethoxysilane (Sigma Aldrich Chemicals, St. Louis, MO, USA) and stained with hematoxylin and eosin, and then histologically examined.

The localization of FasL, Fas, activated caspase-3 and ssDNA (an apoptotic cell marker [15]) in LBs was detected immunohistochemically. Briefly, serial paraffin sections were treated to inactivate endogenous peroxidase by immersion in $0.3 \%(\mathrm{v} /$ v) $\mathrm{H}_{2} \mathrm{O}_{2}$ in methanol and preincubated with $5 \%(\mathrm{v} /$ v) goat normal serum in phospate-buffered saline (PBS; pH 7.4) for $1 \mathrm{~h}$ at RT (20-25 C). They were incubated with each diluted primary antibody: rabbit IgG to mouse-FasL (Santa Cruz Biotechnology, Santa Cruz, CA, USA), rabbit IgG to mouse-Fas (Santa Cruz), rabbit IgG to mouse activated caspase-3 (Cell Signaling Technology, Beverly, MA, USA) and rabbit IgG to ssDNA 
(Dako, Glostrup, Denmark) for $18 \mathrm{~h}$ at $4 \mathrm{C}$, and washed well with PBS. Each antibody was diluted with PBS containing $5 \%(\mathrm{w} / \mathrm{v})$ bovine serum albumin (PBS-BSA) at a concentration of $1 \mu \mathrm{g} / \mathrm{ml}$. For the detection of FasL, Fas and caspase-3, the slides were postincubated with biotinylated secondary antibody to rabbit IgG (Vector, Burlingame, CA, USA) for $30 \mathrm{~min}$ at RT, washed with PBS, and treated with the horseradish peroxidase (HRP)-conjugated avidin-biotin complex antibody (Vector) for $1 \mathrm{~h}$ at RT. For the detection of ssDNA, the slides were postincubated with HRP-labeled secondary antibody to rabbit IgG (Dako envision rabbit link; Dako) for $60 \mathrm{~min}$ at RT. After a wash with PBS, the sections were immersed in $50 \mathrm{mM}$ Tris- $\mathrm{HCl}$ ( $\mathrm{pH} 7.2)$ containing $0.05 \%(\mathrm{w} / \mathrm{v})$ 3,3 diaminobenzidine and $0.002 \%(\mathrm{v} / \mathrm{v}) \mathrm{H}_{2} \mathrm{O}_{2}$ for 1 min at RT. After a wash with distilled water, they were counterstained with methylgreen, washed with distilled water, dehydrated, mounted with Entellan (Merck, Darmstadt, Germany), and examined by light microscopy.

The slides were examined under a microscope. As negative controls, sections incubated without primary and/or secondary antibodies were prepared in each experimental run. As physiological positive controls for ssDNA staining, paraffin sections prepared from jejunums and testes of mature male mice were used.

\section{RT-PCR analyses for FasL, Fas, caspase- 8 and caspase-3}

As described previously $[16,17]$, for RT-PCR analysis, total RNA was extracted from each ovary with a RNeasy mini kit (Qiagen, Chatsworth, CA, USA), treated with a RNase-free DNase kit (Qiagen) to eliminate residual genomic DNA, and then reverse-transcribed with a T-primed firststrand kit (Amersham Pharmacia Biotech, Piscataway, NJ, USA) to synthesize cDNA. Primer pairs specific for partial cDNA sequences of FasL were as follows, forward: 5'-TCCAG GGTGG GTCTA CTTAC TAC-3' and reverse: 5'-CCCTC TTACT TCTCC GTTAG GA-3'; expected PCR product size, $200 \mathrm{bp}$. Those for Fas were, forward: 5'-TGCAG TTGCT GAGAT GAACC-3' and reverse: 5'-GGAAG GTCTT CAATT AACTG CG3'; expected PCR product size, $189 \mathrm{bp}$. Those for caspase- 8 were, forward: 5'-GTGAA GAACT GCGTT TCCTA CC-3' and reverse: 5'-AGCTT CTTCC GTAGT GTGAA GG-3'; expected PCR product size, $206 \mathrm{bp}$. Those for caspase-3 were, forward: 5'-TGTCA TCTCG CTCTG GTACG-3' and reverse: 5'-AAATG ACCCC TTCAT CACCA3'; expected PCR product size, 199 bp. Glyceraldehydes-3-phosphate dehydrogenase (GAPDH; GeneBank accession number M32599, used as an intrinsic control) was amplified with the following primers, forward: 5'-AACGA CCCCT TCATT GAC-3' and reverse: 5'-TCCAC GACAT ACTCA GCAC-3'; expected PCR product size, 191 bp. PCR amplification was performed as follows: platinum Taq DNA polymerase (10,000 Units/ml; Gibco BRL, Grand Island, NY, USA) was added to the cDNA mixture and denatured. The mixture was subjected to PCR in a thermal cycler (GeneAmp PCR Systems 2400; PE Applied Biosystems, Foster City, CA, USA). The hot-start PCR cycles for FasL, Fas, caspase- 8 and GAPDH were as follows: $94 \mathrm{C}$ for $1 \mathrm{~min}$, and then 30 cycles of $94 \mathrm{C}$ for $1 \mathrm{~min}-59 \mathrm{C}$ for $1 \mathrm{~min}-72 \mathrm{C}$ for $1 \mathrm{~min}$, final extension at $72 \mathrm{C}$ for $5 \mathrm{~min}$. The PCR cycles for caspase- 3 were as follows: $94 \mathrm{C}$ for $45 \mathrm{sec}$, and then 30 cycles of $94 \mathrm{C}$ for $1 \mathrm{~min}-61 \mathrm{C}$ for $45 \mathrm{sec}-72 \mathrm{C}$ for 1 min, final extension at $72 \mathrm{C}$ for $5 \mathrm{~min}$. PCR products were electrophoresed in $2 \%(\mathrm{w} / \mathrm{v})$ agarose gels (Sigma) and stained with ethidium bromide (Wako Pure Chemicals, Osaka, Japan). A ready-load $100 \mathrm{bp}$ DNA ladder (Gibco) was used as a molecular weight marker for electrophoresis. After electrophoresis, the stained gels were recorded with a digital fluorescence-recorder (LAS1000; Fuji Film, Tokyo, Japan), and the mRNA expression level, the fluorescence intensity of each band of RCR product, was quantified with ImageGauge (Fuji Film) on a Macintosh computer. The relative abundance of specific mRNA was normalized to the relative abundance of GAPDH mRNA.

\section{Western immunoblotting}

The protein fraction was prepared from each tissue sample as previously reported [16, 17]. Briefly, each sample was homogenized in $60 \mathrm{mM}$ Tris- $\mathrm{HCl}, \mathrm{pH} 6.8$, containing $2.5 \%(\mathrm{w} / \mathrm{v})$ sodium dodecyl sulfate (SDS; Wako), $720 \mathrm{mM} \beta$ mercaptoethanol (Wako) and $1.5 \%(\mathrm{w} / \mathrm{v})$ bromophenol blue (Wako), and then the homogenized sample was mixed with an equal volume of $20 \mathrm{mM}$ Tris- $\mathrm{HCl}, \mathrm{pH} 7.5$, containing 138 $\mathrm{mM} \mathrm{NaCl}, 10 \%$ (v/v) Glycerol, 1\% (v/v) IGEPAL (Sigma), $2 \mathrm{mM}$ ethylenediaminetetraacetic acid 
disodium salt (Wako), $2.1 \mathrm{mg} / \mathrm{ml}$ aprotinin (Sigma), $1 \mu \mathrm{g} / \mathrm{ml}$ leupeptin (Sigma), $1 \mu \mathrm{g} / \mathrm{ml}$ pepstatin A (Sigma), $0.25 \%(\mathrm{w} / \mathrm{v})$ sodium deoxycholate (Wako) and $1 \mathrm{mM}$ pefabloc-SC (Roche Diagnostics, Mannheim, Germany) and boiled for $5 \mathrm{~min}$. The protein concentration in each mixture was measured with a Bradford kit (Wako), and then the protein fraction $(20 \mu \mathrm{g} /$ lane) prepared from each sample was separated by $7.5 \%$ SDSpolyacrylamide gel electrophoresis (SDS-PAGE), and then transferred onto nitrocellulose membranes (Immobilon-P membranes; Millipore, Bedford, MA, USA). The membranes were stained with $0.2 \%(\mathrm{w} / \mathrm{v})$ Ponceau S solution (Serva Electrophoresis, Heidelberg, Germany), and immersed in blocking solution $(100 \mathrm{mM}$ Tris $\mathrm{HCl}$, $\mathrm{pH} 7.6,50 \mathrm{mg} / \mathrm{ml}$ skim milk, $50 \mathrm{mM} \mathrm{NaCl}$ and 1 $\mathrm{mg} / \mathrm{ml}$ Tween 20; Sigma) for $1 \mathrm{~h}$ at room temperature, and then incubated with rabbit antiactivated caspase- 3 polyclonal antibody (Cell Signaling technology, Beverly, MA, USA; 1/200 dilution with blocking solution) for $18 \mathrm{~h}$ at $4 \mathrm{C}$. After being washed with washing solution $(10 \mathrm{mM}$ Tris- $\mathrm{HCl}, \mathrm{pH} 7.6,100 \mathrm{mM} \mathrm{NaCl}$ and $1 \mathrm{mg} / \mathrm{ml}$ Tween 20), they were incubated with horseradish peroxidase-conjugated goat anti-rabbit IgG antibody (Dako; 1/2,000 dilution with washing solution containing $50 \mathrm{mg} / \mathrm{ml}$ skim milk) for $1 \mathrm{~h}$ at room temperature, and then chemiluminescence was visualized with an ECL system (Amersham) according to the manufacturer's protocol. The chemiluminescence was recorded with a digital recorder and then protein expression levels (arbitrary units) were quantified with ImageGauge on a Macintosh computer.

\section{Results}

RT-PCR for FasL, Fas, caspase-8 and caspase-3 $m R N A s$ and Western immunoblotting for activated caspase-3

The mRNAs of FasL, Fas, caspase-8, caspase- 3 and GAPDH were detected in the ovaries of SAMR and SAMP mice at the P, E, M and D stages (Fig. 1A). In SAMR ovaries at the D stage, higher expression levels of FasL, Fas and caspase- 8 mRNAs were observed (Fig. 1B), but higher levels were found in SAMP ovaries at the M stage. Higher expression levels of caspase- 3 in SAMR ovaries were seen at the $\mathrm{P}$ and $\mathrm{M}$ stages, but those in SAMP ovaries were noted at the E and D stages.

Western immunoblotting showed a higher expression level of activated caspase-3, a $17 \mathrm{kDa}$ fragment, in SAMR ovaries at the $\mathrm{P}$ stage when compared with SAMP ovaries (Fig. 1C and D). Trace amounts of activated caspase- 3 were detected in SAMR and SAMP ovaries at the E, M and D stages.

\section{Histology and immunohistochemistry for ssDNA, FasL, Fas and activated caspase-3}

More than 50 LBs/ovary were observed in the ovaries of SAMP ovaries (Fig. 2A), and less than 20 LBs/ovary were seen in SAMR mice (Fig. 2F), indicating more than 30 LBs/ovary are aaLBs in SAMP ovaries. No marked difference in the number of LBs was seen between the ovaries of SAMR mice and age-matched ICR mice (less than 20 LBs/ovary).

At the P stage, many ssDNA-positive/apoptotic cells were detected in luteal cells in the ovaries of SAMR mice (Fig. 2B), but no apoptotic cell was seen at the E, M or D stages. Notably, no apoptotic cell was found in aaLBs in the ovaries of SAMP at the P, E, $M$ or D stages (Fig. 2G). In the ovaries of SAMR mice at the $\mathrm{P}$ stage, strong immunostaining for of FasL and Fas proteins was observed in some luteal cells of newly formed LBs and in most luteal cells of regressing LBs (Fig. 2C and D, respectively). In SAMP ovaries at the $\mathrm{P}$ stage, similar results were obtained in regressing LBs, but aaLBs showed negative staining for FasL or Fas protein (Fig. $2 \mathrm{H}$ and I, respectively). The localization of Fas protein corresponded with that of FasL protein. Activated caspase-3 protein was detected in luteal cells of regressing LBs of both SAMR and SAMP ovaries at the P stage (Fig. 2E and J, respectively), but not in those of aaLBs of SAMP ovaries.

\section{Discussion}

The life span of SAMP mice is shorter than that of other mouse models used for aging research (CBA, C57BL, RFM and NZB strains) [3], and agedependent changes in reproductive functions are more severe in SAMP mice than in other murine models [4-7]. Previously, we found that some LBs in the ovaries of SAMP mice with regular estrus cycles were abnormally accumulated [8]. Because $\mathrm{P} 4$ is synthesized and then rapidly catabolized into 

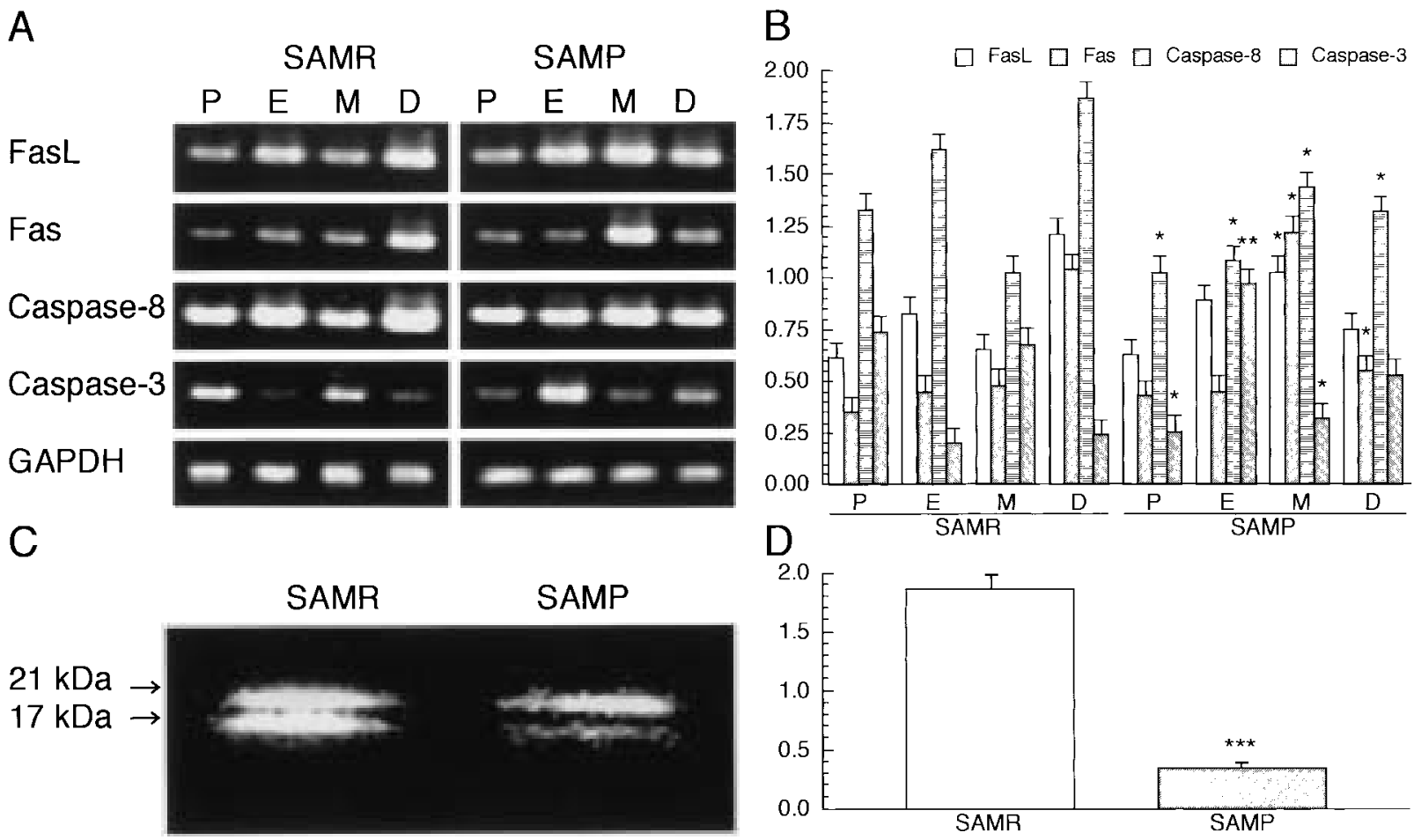

Fig. 1. Representative photographs of RT-PCR products for FasL, Fas, caspase-8, caspase-3 and GAPDH mRNAs in ovaries of SAMR and SAMP at the proestrus (P), estrus (E), metestrus (M) and diestrus (D) stages are shown in A. Their mRNA expression levels (FasL, Fas, caspase- 8 or caspase- 3 mRNA/GAPDH mRNA ratio) in ovaries are shown in B. Representative photographs of chemiluminescence-Western immunoblotting for activated caspase-3 protein, $17 \mathrm{kDa}$ bands, in ovaries of SAMR and SAMP at the proestrus stage are shown in C. Activated caspase-3 protein was quantified by means of an image-analyzer and shown as chemiluminescence levels (D). All data in B and D are shown as means \pm SEM. ${ }^{*}, * *$ and ${ }^{* * *}: \mathrm{P}<0.05,0.01$ and 0.001 vs each SAMR sample.

an inactive $20 \alpha$-hydroxyprogesterone in luteal cells of aaLBs in SAMP ovaries (functional regression occurs), SAMP mice can show normal consecutive 4-day estrus cycles. In such aaLBs of SAMP mice, no apoptotic cells were detected histochemically, but many apoptotic cells were detected in the regressing LBs of SAMP ovaries. These findings confirmed that an arrest of morphological regression (deficiency of apoptotic cell death in luteal cells) occurs in the luteal cells of aaLBs in SAMP mice. Because previous studies showed that the FasL/Fas system plays a crucial role in luteolysis [12,13], we carried out the present experiments. We showed abnormal expression patterns of the mRNAs of FasL/Fas-mediated apoptosis signaling molecules (FasL, Fas, caspase-8 and caspase-3) and of activated caspase- 3 protein in whole ovarian tissue samples of SAMP mice compared with those of SAMR mice. Moreover, apoptotic luteal cells were demonstrated in regressing LBs of the ovaries of SAMR and SAMP mice at the $P$ stage but not the E, M or D stages, and no apoptotic luteal cell was detected in aaBLs of SAMP mice during the estrous cycle. In both SAMR and SAMP mice, most of the luteal cells of regressing LBs produce FasL and Fas proteins, but the luteal cells of aaLBs in SAMP mice do not produce them. Moreover, activated caspase- 3 protein was not demonstrated in the luteal cells of aaLBs of SAMP ovaries. In conclusion, a deficiency of apoptosis signaling molecules in luteal cells of aaLBs in SAMP mice causes the arrest of morphological regression in luteal cells, resulting in the abnormal accumulation of LBs. In the future, we have to elucidate which factor(s) induce the deficiency of FasL/Fas-mediated apoptosis signaling molecules in luteal cells of aaLBs in SAMP mice. 


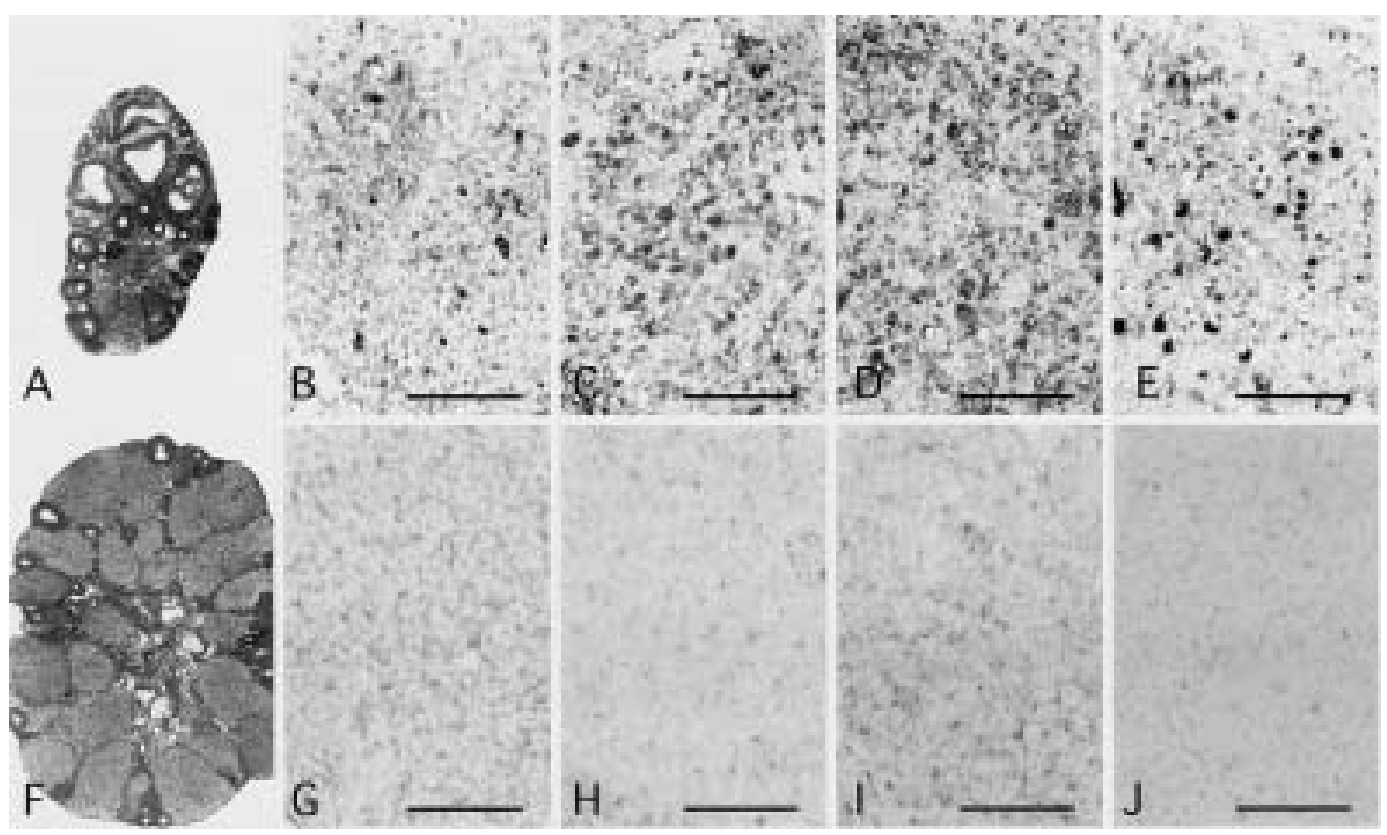

Fig. 2. Ovarian sections from 24-week-old SAMR (A, B, C, D and E) and SAMP (F, G, H, I and J) mice at the proestrus stage. Hematoxylin and eosin staining was performed for histological assessment (A and F) Apoptotic cells were detected immunohistochemically in luteal bodies with anti-single strand DNA (ssDNA) antibody (B and G). Representative sections of luteal bodies were subjected to immunohistochemistry for FasL (C and H), Fas (D and I) and activated caspase-3 (E and J) proteins. Scale bars in B-E and G-J indicate $100 \mu \mathrm{m}$.

\section{Acknowledgements}

This work was supported by Grants-in-Aid for Creative Scientific Research (13GS0008) and for
Scientific Research on Priority Areas (A) (13027241) to N. M. from the Ministry of Education, Culture, Sports, Science and Technology in Japan.

\section{References}

1. Takeda $T$, Hosokawa $M$, Takeshita $S$, Irino $M$, Higuchi K, Matsushita T, Tomita Y, Yasuhira K, Hamamoto H, Shimizu K, Ishii M, Yamamuro T. A new model of accelerated senescence. Mech Aging Dev 1981; 17: 183-194.

2. Takeda T, Hosokawa M, Higuchi K. Senescenceaccelerated mouse (SAM): a novel murine model of accelerated senescence. J Am Geriat Soci 1991; 39: 911-919.

3. Masoro EJ. Animal models in aging research. In: Schneider EL, Rowe JW (eds.), Handbook of the Biology of Aging. New York: Academic Press; 1990: 72-94.

4. Miyamoto H, Manabe N, Akiyama Y, Mitani Y, Sugimoto $\mathbf{M}$, Sato E. Quantitative studies on spermatogenesis in the senescence accelerated mouse. In: Takeda T, Hosokawa M (eds.), The SAM Model. Amsterdam: Elsevier Sci Pub; 1994: 275-278.
5. Miyamoto H, Manabe N, Akiyama $\mathbf{Y}$, Watanabe T, Sugimoto M, Sato E. A morphometric study of spermatogenesis in the testes of mice of a senescence accelerated strain. Experientia 1994; 50: 808-811.

6. Miyamoto $\mathbf{H}$, Manabe $\mathrm{N}$, Watanabe $\mathrm{T}$, Aruga $\mathrm{C}$, Mitani Y, Sugimoto N, Sugimoto M, Sato E. Female reproductive characteristics of the senescence accelerated mouse. In: Takeda $\mathrm{T}$, Hosokawa M (eds.), The SAM Model. Amsterdam: Elsevier Sci Pub; 1994: 279-282.

7. Miyamoto H, Manabe N, Mitani Y, Sugimoto N, Watanabe T, Aruga C, Sato E. Female reproductive properties and prenatal development of a senescence accelerated mouse strain. J Exp Zool 1995; 272: 116-122.

8. Kiso M, Manabe N, Komatsu K, Nisioka N, NakaiSugimoto N, Miyamoto $\mathbf{H}$. Abnormal accumulation of luteal bodies in ovaries of the senescence 
accelerated mouse (SAM). J Reprod Devel 2001; 47: 153-158.

9. Sakamaki K, Yoshida H, Nishimura $Y$, Nishikawa $\mathrm{S}$, Manabe $\mathbf{N}$ and Yonehara $\mathbf{S}$. Involvement of Fas in ovarian follicular atresia and luteolysis. Mol Reprod Dev 1997; 47: 11-18.

10. Quirk SM, Harman RM, Huber SC, Cowan RG. Responsiveness of mouse corpora luteal cells to Fas antigen (CD95)-mediated apoptosis. Biol Reprod 2000; 63: 49-56.

11. Cohen PL, Eisenberg RA. Lpr and gld: single gene models of systemic autoimmunity and lymphoproliferative disease. Annu Rev Immunol 1991; 9: 243-269.

12. Komatsu K, Manabe N, KisDo M, Shimabe M, Miyamoto $\mathbf{H}$. Changes in localization of immune cells and cytokines in corpora lutea during luteolysis in murine ovaries. J Exp Zool 2003; 296: 152-159.

13. Komatsu K, Manabe N, KisDo M, Shimabe M, Miyamoto H. Soluble Fas (FasB) regulates luteal cell apoptosis during luteolysis in murine ovaries. Mol
Reprod Dev 2003; 65: 345-352.

14. Nagata S. Apoptosis by death factor. Cell 1997; 88: 355-365.

15. Watanabe I, Toyoda M, Okuda J, Tenjo T, Tanaka K, Yamamoto $T$, Kawasaki $H$, Sugiyama $T$, Kawarada Y, Tanigawa N. Detection of apoptotic cells in human colorectal cancer by two different in situ methods: antibody against single stranded DNA and terminal deoxynucleotidyl transferase mediated dUTP-biotin nick end-labeling (TUNEL) methods. Jpn J Canc Res 1999; 90: 188-193.

16. Nakayama $\mathbf{M}$, Manabe $\mathbf{N}$, Inoue $\mathbf{N}$, Matsui $T$, Miyamoto $\mathbf{H}$. Changes in the expression of tumor necrosis factor (TNF) $\alpha, \mathrm{TNF} \alpha$ Receptor (TNFR) 2 and TNFR-associated factor 2 in granulosa cells during atresia in pig ovaries. Biol Reprod 2003; 68: 530-535.

17. Matsui T, Manabe N, Goto $\mathrm{Y}$, Inoue N, Nishihara $\mathbf{S}$, Miyamoto $\mathbf{H}$. Changes in the expression and activity of caspase- 9 and Apaf1 in granulosa cells during follicular atresia in pig ovaries. Reproduction 2003; 125: 823-830. 